\title{
Measurement of partial atomic charges by least-squares refinement of variable electron density crystallographic models.
}

\author{
M Zdilla ${ }^{1}$, T Keller $^{2}$, P Prakash ${ }^{3}$, A Byrne $^{2}$ \\ ${ }^{1}$ Chemistry, Temple Univ, Philadelphia, PA, ${ }^{2}$ Temple University, Philadelphia, PA, ${ }^{3}$ IISER Pune, \\ Pune, Maharashtra \\ mzdilla@temple.edu
}

Of interest in understanding electronic structure, bulk physical properties, enthalpies of phase changes, dipole moments, and numerous other properties of molecules, is the determination of realistic partial atomic charges on atoms. Atoms may take on partial positive or negative charges due to polar covalent bonds, coordinate covalent bonds, or due to formal charges imposed by Lewis structure constraints. Traditional crystallographic refinement treats each atom as a neutral, spherical atom however. We present a ongoing developments of a mode of crystallographic model refinement that permits refinement of electron density at individual atoms in order to arrive at partial atomic charges of atoms in a crystallographic model. Comparison to calculated partial charges (CHELPG, NBO, Mulliken) from quantum calculations (DFT, MP2) in both the gas phase and crystalline state will be presented.

Acta Cryst. (2020). A76, a152 